Supporting Information

\title{
Toward Near-Perfect Diffractive Optical Elements via Nanoscale 3D Printing
}

\author{
Hao Wang, ${ }^{1}$ Hongtao Wang, ${ }^{1}$ Wang Zhang, ${ }^{1}$ Joel K.W. Yang ${ }^{1,2, ~ *}$
}

${ }^{1}$ Engineering Product Development Pillar, Singapore University of Technology and Design, 8 Somapah Road, Singapore 487372, Singapore

${ }^{2}$ Institute of Materials Research and Engineering, A*STAR (Agency for Science, Technology and Research), 2 Fusionopolis Way, \#08-03 Innovis, 138634 Singapore

*Corresponding author: joel_yang@sutd.edu.sg 


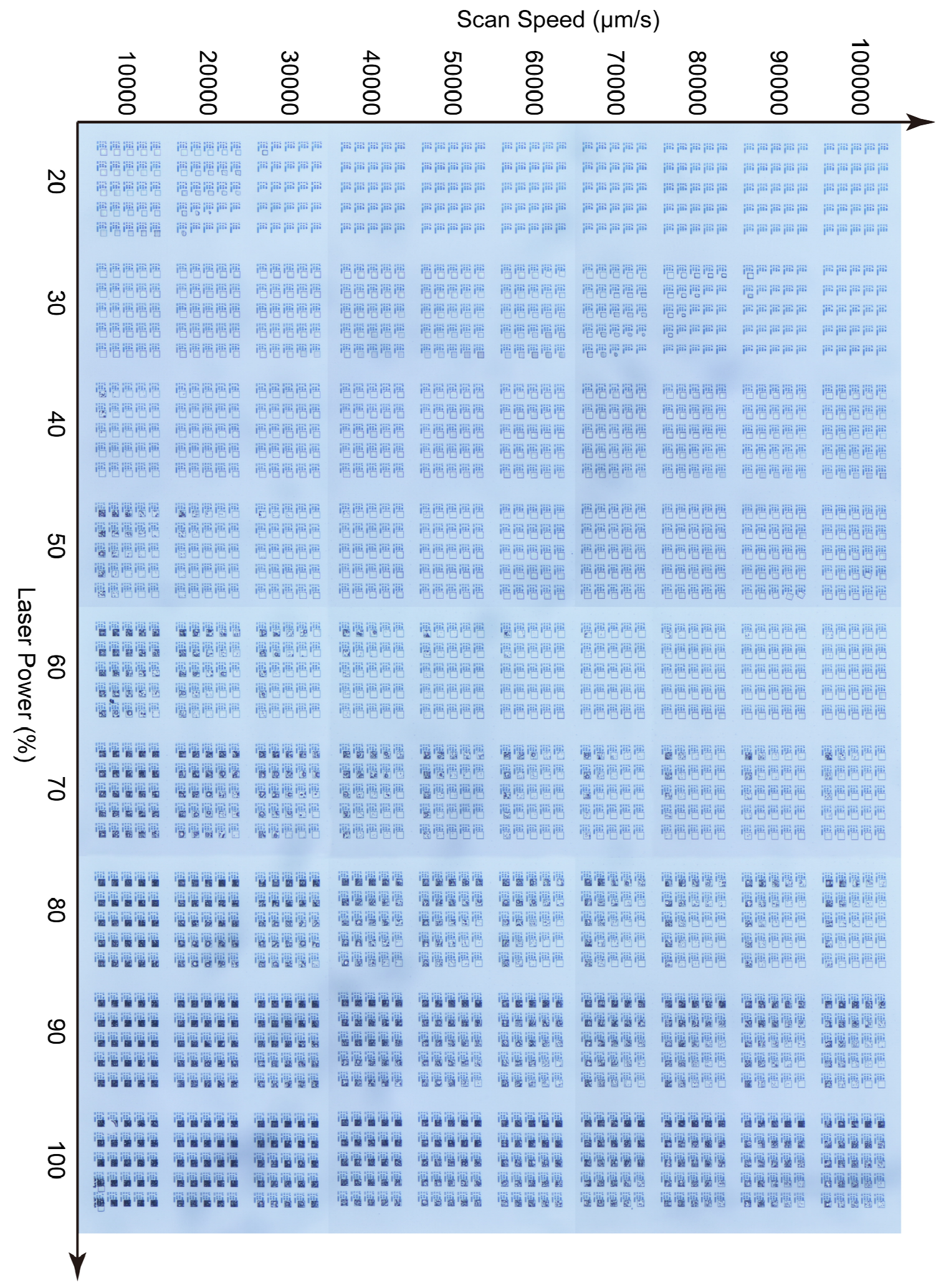

Figure S1. Phase block array printing by nest sweep of the 3D printing parameters with two- 
photon polymerization lithography. For the outer printing parameters, the ratio of laser power ranges from $20 \%$ to $100 \%$ with steps of $10 \%$ (total laser power $50 \mathrm{~mW}$ ), and the scan speed from 10000 to 100000 with steps of $10000(\mu \mathrm{m} / \mathrm{s})$. For the inner printing parameters, the hatching distance ranges from 0.10 to $0.30 \mu \mathrm{m}$ with steps of $0.05 \mu \mathrm{m}$ along $x$-axis, and the slicing distance from 0.10 to $0.50 \mu \mathrm{m}$ with steps of $0.10 \mu \mathrm{m}$ along $y$-axis. Each printed phase block is with size $25 \times 25 \times 5 \mu \mathrm{m}^{3}$. At the left-up corner of the printed arrays, most of the blocks are detached from the substrate due to low laser power and limited numbers of laser pulses for polymerization, while at the right-down corner of the printed arrays, the blocks are over-burnt because of the excess power is absorbed by the photoresist. The absorbed energy is beyond the energy threshold and bubbles are generated during printing. The optimized combination of the printing parameters can be found in the middle part of the array by considering the shrinkage, inner defect, printing time, and transparence.
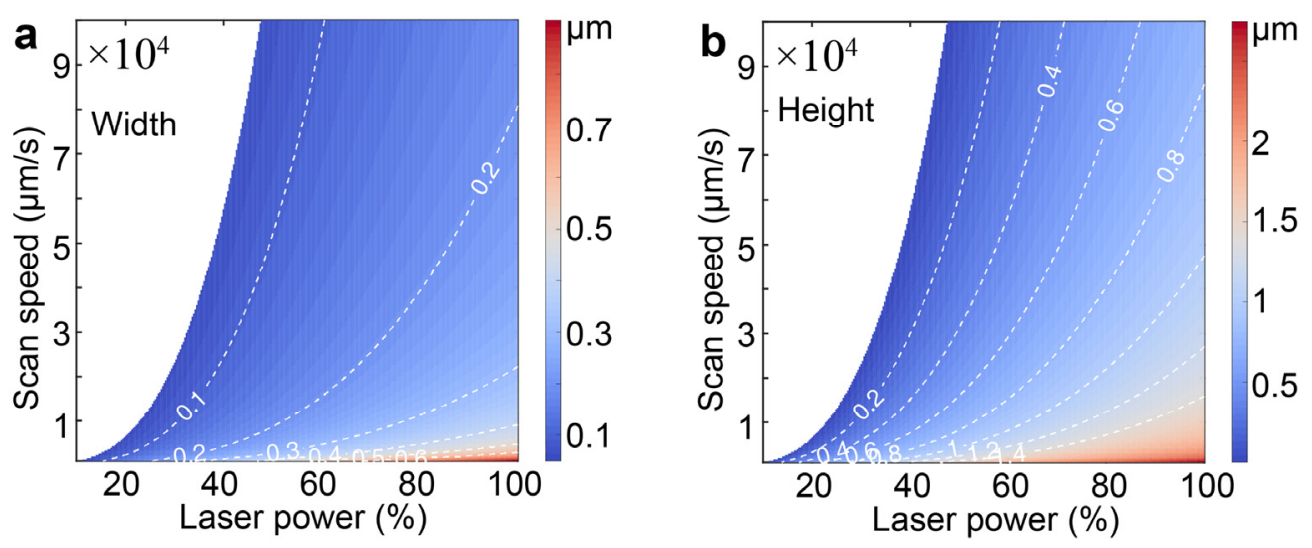

Figure S2. Calcutated width (a) and height (b) of the printed lines with photoresist IP-Dip at different laser power ratios and scan speeds of laser beam with the fitted parameters (total laser 
power $50 \mathrm{~mW})$.

Notes S1 Lumped 3D printing parametric model for two-photon polymerization lithography

Table S1 3D printing parameters (scan speed and ratio of laser power) for line dimensions

\begin{tabular}{ccc}
\hline Array & Scan speed $(\mu \mathrm{m} / \mathrm{s})$ & Ratio of laser power $(\%)$ \\
\hline$A$ & $5,10,20,50$ & $5: 5: 50$ \\
$B$ & $100: 100: 900$ & $10: 10: 100$ \\
$C$ & $1000: 1000: 10000$ & $10: 10: 100$ \\
$D$ & $15000: 5000: 45000$ & $40: 10: 130$ \\
$E$ & $50000: 10000: 100000$ & $40: 10: 130$ \\
\hline
\end{tabular}

First, a semiempirical analytic model fitted from the experimental data and simulation can be used to predict the line dimensions of two-photon polymerized IP-Dip in Nanoscribe at a large range of laser power and scan speed of the femtosecond laser beam. ${ }^{1}$ The experimental data of width and height of the printed lines via ascending scan method can be obtained from the SEM images. The fitted parametric models for line dimensions can be written as ${ }^{1}$

$$
\left\{\begin{array}{l}
W=\left(\alpha_{W}(S) P^{\beta_{W}}+\gamma_{W}\right)\left(1+\varsigma_{W} S^{\chi_{W}} P\right) \\
H=\left(\alpha_{H}(S) P^{\beta_{H}}+\gamma_{H}\right)\left(1+\varsigma_{H} S^{\chi_{H}} P\right)
\end{array}\right.
$$

where $W$ and $H$ represent the width and height of the printed lines, respectively, $S$ is the effective scan speed with unit $\mu \mathrm{m} / \mathrm{s}, P$ is the laser power ratio with the total laser power $50 \mathrm{~mW}, \alpha, \beta, \gamma, \varsigma$, and $\chi$ are modeling coefficients. The relation between $\alpha$ and effective scan speed can also be expressed as

$$
\left\{\begin{array}{l}
\alpha_{W}(S)=\alpha_{W 1} S^{\alpha_{W 2}} \\
\alpha_{H}(S)=\alpha_{H 1} S^{\alpha_{H 2}}
\end{array}\right.
$$

To include the saturation effect of line dimensions at slow scan speed, the effective scan speed is defined as following, 


$$
S=\sqrt{S_{0}^{2}+S_{s a t}^{2}}
$$

in which $S_{0}$ is the actual scan speed during printing and $S_{\text {sat }}$ is the saturation scan speed. The fitted values for the coefficients can be found in Table S2. With this model, different values of laser power and scan speed can be chosen to print lines with the same dimension, hence one can increase the scan speed with adjusted laser power to decrease the printing time.

Table S2 Coefficients of the line dimensions of TPL-printed IP-Dip with Nanoscribe

\begin{tabular}{ccc}
\hline Coefficient & Width $(W)$ & Height $(H)$ \\
\hline$\alpha_{1}$ & 0.370 & -1.984 \\
$\alpha_{2}$ & -0.075 & 0.138 \\
$\beta$ & 0.276 & -0.437 \\
$\gamma$ & -0.384 & 1.799 \\
$\varsigma$ & 0.346 & 0.074 \\
$\chi$ & -0.485 & -0.234 \\
$S_{\text {sat }}$ & 55 & 18 \\
\hline
\end{tabular}

Second, a lumped parameter model can be developed based on the actual physical process to model the proximity effect in two-photon polymerization lithography printed IP-Dip. ${ }^{2}$ As the laser damage occurs when the energy absorbed by the photoresist exceeds the energy threshold, both the single-photon absorption, which comes from the cured resin, and two-photon absorption during polymerization should be considered. With the classical Bee-Lambert absorption relationship, the absorbed energy per unit length $E(\mathrm{~J} / \mathrm{m})$ along the printing direction in the photoresist can be written as ${ }^{2}$

$$
E=\sqrt{\frac{\pi}{2}} \delta_{a}\left(\frac{t f \sigma^{2}}{S_{0}}\right)\left(\bar{\alpha}_{s} \frac{2 \bar{P}}{\pi \sigma^{2}}+\frac{\bar{\alpha}_{t}}{\sqrt{2}}\left(\frac{2 \bar{P}}{\pi \sigma^{2}}\right)^{2}\right)
$$

here $\delta_{\alpha}$ is the characteristic absorption length along the axial direction, $t$ is the duration of a single 
pulse, $f$ is the frequency of pulse, $\sigma$ is the beam radius at the focal plane, $S_{0}$ is the actual scan speed in printing, $\bar{\alpha}_{s}, \bar{\alpha}_{t}, \bar{P}$ are the area-averaged single-photon and two-photon absorptivity, timeaveraged power of a single pulse, respectively. To avoid the bubble generation during printing, the absorbed energy should not exceed the energy threshold of the photoresist boiling. Thus we have

$$
E \leq \rho H_{e n t h} \sigma \delta_{t h}
$$

in which $\rho$ is the mass density, $H_{\text {enth }}$ is the equivalent enthalpy of vaporization of photoresist, $\delta_{t h}$ is the thermal characteristic length.

For a solid phase block printed with line by line in $x y$ plane, and layer by layer in $z$ direction, the influence of hatching distance and slicing distance should also be included in the absorption, i.e., the absorption of cured photoresist needs to be taken into consideration. Therefore, the singlephoton absorption can be evaluated as

$$
\begin{aligned}
\bar{\alpha}_{s} & \approx \alpha_{s}\left(\operatorname{erf}\left(\sqrt{2}\left(L_{H}+\frac{W}{2}\right) / \sigma\right)-\operatorname{erf}\left(\sqrt{2}\left(L_{H}-\frac{W}{2}\right) / \sigma\right)\right) \\
& +\alpha_{s}\left(\operatorname{erf}\left(\sqrt{2}\left(L_{S}+\frac{H}{2}\right) / \sigma^{\prime}\right)-\operatorname{erf}\left(\sqrt{2}\left(L_{S}-\frac{H}{2}\right) / \sigma^{\prime}\right)\right)
\end{aligned}
$$

here $\alpha_{s}=2.22 \mathrm{~cm}^{-1}$ is the single-photon absorptivity, ${ }^{2} L_{H}$ is the hatching distance in the $x y$ plane and $L_{S}$ is the slicing distance along the $z$ direction, $\sigma^{\prime}$ is the beam radius at the axial focal plane, erf represents the Gaussian error function. Thus, with the equations listed above, the relations between power threshold $\bar{P}$ and laser power ratio $P$, scan speed $S_{0}$, slicing distance $L_{S}$ and hatching distance $L_{H}$ can be evaluated.

With optimized printing parameters: scan speed at $40000 \mu \mathrm{m} / \mathrm{s}$ and the ratio of laser power at $50 \%$ (total laser power $50 \mathrm{~mW}$ ), the width and height of printed line can be estimated as $121 \mathrm{~nm}$ 
and $327 \mathrm{~nm}$ by Eq. (1-3) and Table S1. According to the relation between Gaussian beam waist and numerical aperture, $\sigma=0.61 \lambda / \mathrm{NA}$, and $\sigma^{\prime}=2 \lambda / \mathrm{NA}^{2}$, the waist in $x y$ plane can be evaluated as $340 \mathrm{~nm}$, and in the $z$ direction $796 \mathrm{~nm}$. Thus, the single-photon absorption can be evaluated to select the hatching and slicing distances, as seen from Figure $3 \mathrm{~b}$ and $\mathrm{c}$ in the main text. Other examples are shown in Figure S3.
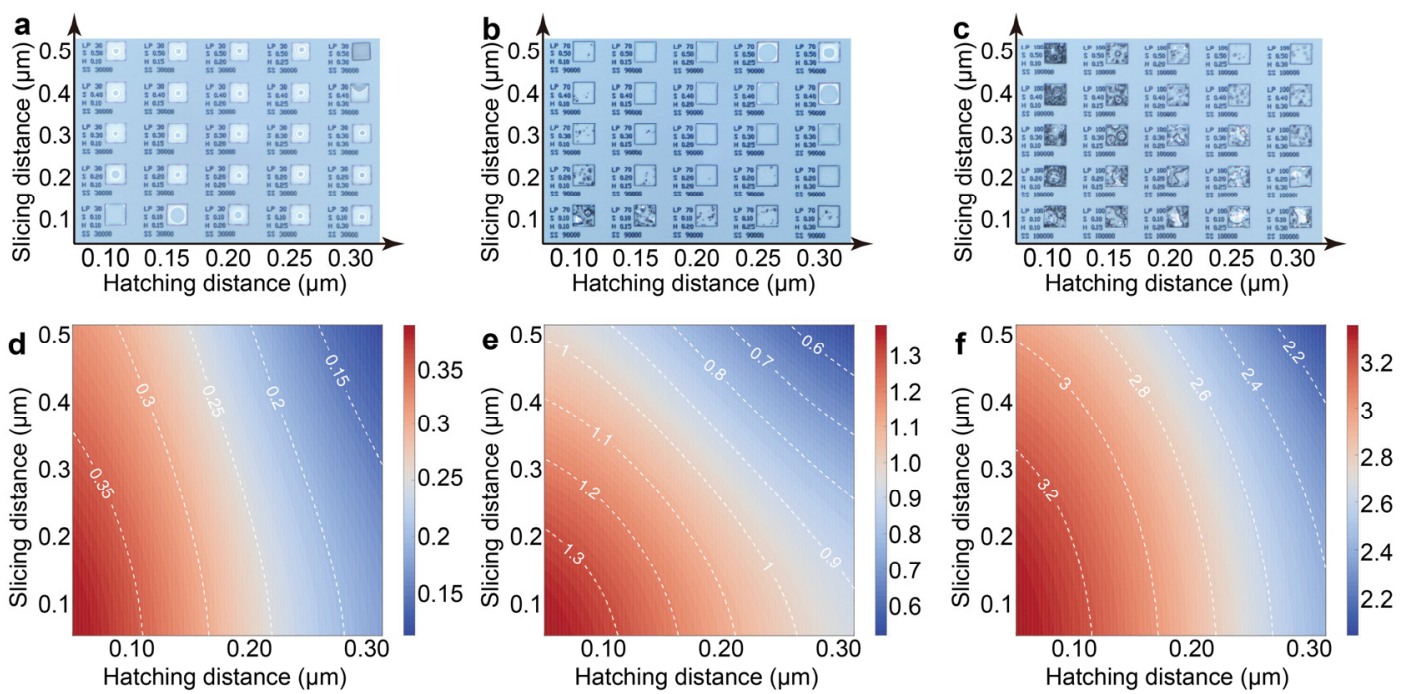

Figure S3. Dependence of single-photon absorption of IP-Dip with hatching and slicing distances in two-photon polymerization lithography. Phase blocks printed with printing parameters: (a) scan speed at $30000 \mu \mathrm{m} / \mathrm{s}$ and the ratio of laser power at $30 \%$ (total laser power $50 \mathrm{~mW}$ ), (b) scan speed at $90000 \mu \mathrm{m} / \mathrm{s}$ and the ratio of laser power at 70\%, (c) scan speed at $100000 \mu \mathrm{m} / \mathrm{s}$ and the ratio of laser power at $100 \%$. The hatching distance ranges from 0.10 to $0.30 \mu \mathrm{m}$ with steps of $0.05 \mu \mathrm{m}$ along $x$-axis, and the slicing distance from 0.10 to $0.50 \mu \mathrm{m}$ with steps of $0.10 \mu \mathrm{m}$ along $y$-axis. (d, e) Normalized single-photon absorption (unit: $\alpha_{s}$ ) of 3D-printed phase blocks corresponding to (ac). 

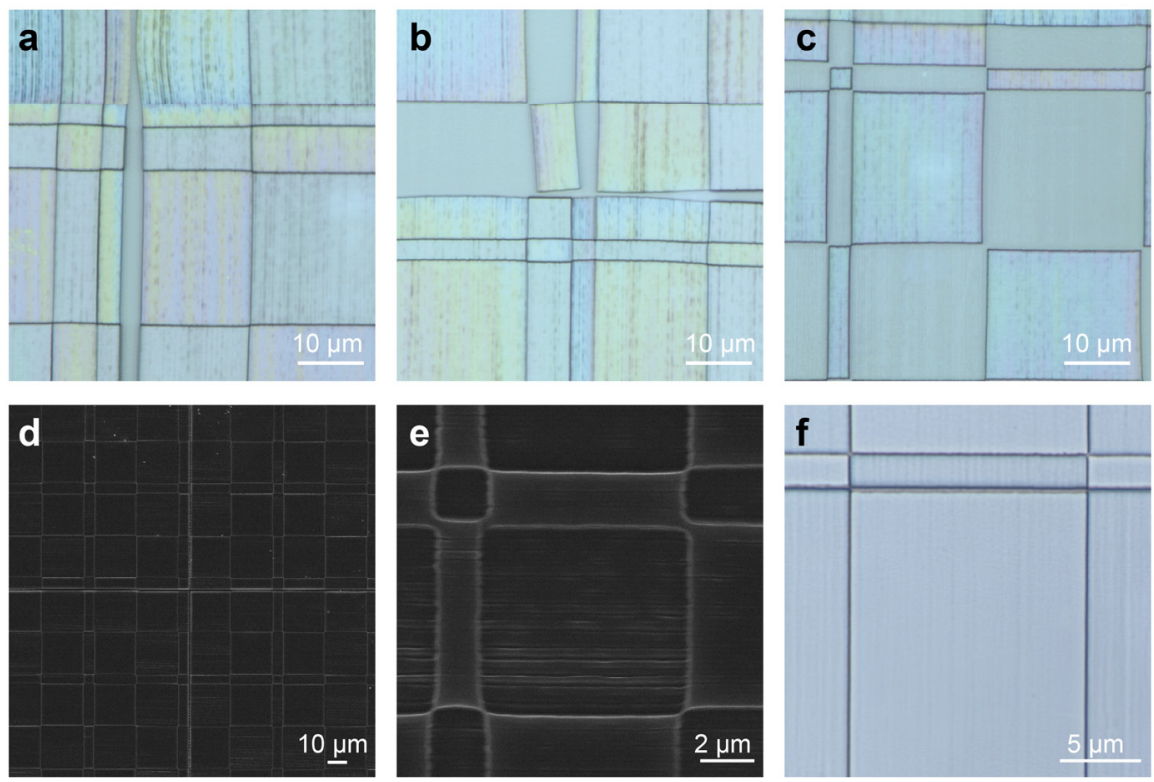

Figure S4. Comparison of the Dammann gratings (DGs) printed by unoptimized and optimized printing parameters. Optical microscopic images (a, c) and SEM images (d, e) of the DGs printed with officially recommended printing parameters: scan speed at $10000 \mu \mathrm{m} / \mathrm{s}$ and the ratio of laser power at $38 \%$ (total laser power $50 \mathrm{~mW}$ ), hatching distance at $0.2 \mu \mathrm{m}$ and slicing distance at 0.29 $\mu \mathrm{m}$. (f) Optical microscopic image of the DGs printed with optimized printing parameters: scan speed at $40000 \mu \mathrm{m} / \mathrm{s}$ and the ratio of laser power at $50 \%$ (total laser power $50 \mathrm{~mW}$ ), hatching distance at $0.1 \mu \mathrm{m}$ and slicing distance at $0.29 \mu \mathrm{m}$. a-e show that there are different types of defects in the unoptimized structures, such as the nonuniform shrinkage of phase blocks, large thickness variation (different colors of different positions of the phase blocks), gaps between adjacent writing fields, displacement of the phase blocks (detached from the substrate), distortion of the straight edges, and disconnection/over-connection between nearby phase blocks. Image $\mathbf{f}$ shows that with the optimized printing parameters, the nonuniform shrinkage is minimized and other defects are 
avoided. The gaps between writing fields stem from the accumulation of movement error of the stage are also minimized and almost cannot be observed. The uniform color of the DGs indicates the high quality of the printed structures.

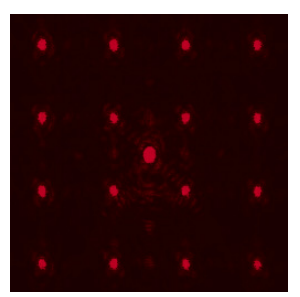

Figure S5. Experimental image of the diffraction spots with the Dammann gratings at order $O=4$ printed by unoptimized printing parameters: scan speed at $10000 \mu \mathrm{m} / \mathrm{s}$ and the ratio of laser power at $38 \%$ (total laser power $50 \mathrm{~mW}$ ), hatching distance at $0.2 \mu \mathrm{m}$ and slicing distance at $0.29 \mu \mathrm{m}$. The measured total diffraction efficiency of the 16 diffraction spots is $38.4 \%$ and the power ratio of the zero order spot is $13.7 \%$. 


\section{Notes S2 Reducing the shrinkage and deformation of printed structures}

The 3D-printed polymer structures generally are not very thermally and mechanically stable, and may lead to deformation due to temperature changes and optical irradiation. The primary cause of the shrinkage and the resulting deformation observed in the final structures are due to low degree of conversion (DC) of resin. The conversion varies across the width of the line, being greatest at the point of peak irradiance at the center of the focal spot and lower near the edge of the Gaussian beam, inducing incomplete photoreaction of the resin. In addition, the subsequent removal of the unreacted resin during development also further deteriorate the final mechanical properties. ${ }^{3}$ Hence with higher DC value, the printed structures will be more stable. Differential scanning calorimetry (DSC), Fourier transform infrared (FTIR) spectroscopy, Raman microspectroscopy, and coherent anti-Stokes Raman scattering (CARS) spectroscopy can be used to detect the DC value of polymerized resins.

For IP series resins from Nanoscribe, the DC value can be calculated from the following equation when using Raman microspectroscopy, ${ }^{4}$

$$
\mathrm{DC}=\left[1-\left(\frac{A_{C=C} / A_{C=\mathrm{O}}}{A_{C=C}^{\prime} / A_{C=\mathrm{O}}^{\prime}}\right)\right] \times 100 \%
$$

In which, $A_{C=C}, A_{C}=O, A^{\prime}{ }^{\prime}=C$, and $A^{\prime}{ }^{\prime}=O$ are the integrated peak intensities of Raman peaks related to the $\mathrm{C}=\mathrm{C}$ and $\mathrm{C}=\mathrm{O}$ moieties in the polymerized and the non-polymerized photoresist. They provide the molecular details about the monomer/oligomer photo-conversion. It has been found that DC shows a stronger dependence on the average laser power than the scan speed of laser beam, the results also confirmed by the nano-indentation measurement. Hence DC value can be used to 
effectively evaluate the mechanical properties of 3D-printed elements by two-photon polymerization lithography (TPL).

It should be noted that DC values of $100 \%$ are not physical possible with the polyfunctional monomers used in most TPL resins. This is due to the formation of highly cross-linked networks that severely restrict functional group mobility. The maximum DC values of IP series photoresists are between 45 and 75\%, ${ }^{5}$ and for 3D-printed IP-Dip elements, the maximum Young's modulus is around $4.5 \mathrm{GPa}^{3}$

There are many methods have been proposed to improve the DC value to enhance the mechanical properties, or reduce the external forces during development to maintain the 3Dprinted structures.

It has been shown that the enhanced local optical fields surrounding nanoparticles ${ }^{6}$ and metallic bowtie nano-antennae ${ }^{7}$ can trigger the photochemical reaction at lower intensities of incident laser beam, or even incoherent light source. ${ }^{6}$ Hence blending nanoparticles into resin could help to increase the DC value thus enhance the mechanical properties. The intrinsic high mechanical strength of the particles, or frames formed by nanowires and nanotubes can further strengthen the toughness of the printed structures. However, there are some disadvantages that should be carefully considered. First, they need to be uniformly mixed with the photoresist. Still, they might still cause local hot spots leading to bubbling even when photoresist at other places is under-polymerized. Second, the nanowires, nanotubes and nanoparticles may cause strong light scattering, reducing the transmittance significantly if not index matched to the cured resist, thus affecting the optical performance of the DOEs. Last, they may also induce greater surface roughness to the DOEs and 
lower the diffraction efficiency. Therefore, the nanoparticles could be blended into the photoresist to enhance the mechanical properties. However, this method may not be a good candidate for diffractive optical applications due to above reasons, especially for high performance DOEs. ${ }^{8}$

Other methods such as modifying the 3D model to compensate for the expected shrinkage needs complex and nonlinear shape compensation processes. ${ }^{9}$ Multipath scanning method (MPS) can be used to reinforce the contour of 3D structures by increasing the effective laser power and exposure time. However, when the laser power and exposure time are increased, the voxel size grows gradually based on the increase of threshold region for polymerization and radical expansion to outside of an initially formed voxel. The height of voxel is also enlarged, thus it is not suitable for an ultraprecise DOE fabrication. ${ }^{10}$ Another suggested method is to use resins that yield polymers with greater strength. For example, custom resins, such as sol-gel resins, with high contents of $\mathrm{Si}-$ $\mathrm{O}$ and/or $\mathrm{Zr}-\mathrm{O}$ content have been used to strengthen printed structures. ${ }^{11}$

Considering the general practicality and effectiveness, a post UV-treatment of reactive groups, which remained unreacted upon the two-photon polymerization lithography, can enhance the cross-linking density. Therefore, the mechanical stability of structures and their resistance against mechanical deformations are improved, with reduced number of defects and collapsed structures. ${ }^{12}$

Furthermore, during development, capillary forces can cause significant defects when the solvent evaporates. In order to reduce capillary forces, liquids with low surface tension and viscosity, such as hexamethyldisilazane and nonafluorobutyl methyl ether are used. ${ }^{12-17}$ In addition, critical point drying with liquid $\mathrm{CO}_{2}$ can also mitigate capillary force effects during drying to help prevent shrinkage and deformation. ${ }^{18}$ 
In our case of study, the printing parameters including laser power, scan speed, hatching distance and slicing distance were optimized to obtain the best printed elements with negligible shrinkage and defects, as shown in Figure 3 and Figure S1. Moreover, the low surface tension liquid nonafluorobutyl methyl ether was used in the last step of post-development to reduce the potential deformation thus maintaining the high performance of our DOEs. 


\section{Notes S3 Uniformity of refractive index of printed phase blocks}

The nonuniformity of polymerization may lead to the nonuniformity of refractive index in the printed elements. However, exposure uniformity is one of the strengths of the Nanoscribe system. The beam position in the horizontal $x y$ plane can be tuned by a group of galvanometric mirrors. Due to the inertia of the mirrors, the writing speed is not constant over a complete trajectory. The mirrors need some time to accelerate to the specified writing speed and some time to decelerate at the trajectory end. Thus the laser power has to be adjusted at the trajectory-starts and at trajectoryends to avoid overexposure at the segments where the writing speed is slower than specified, i.e., at sharp turns and reversal in direction. This laser power adjustment is fully automated and integrated into NanoWrite (the software to control Nanoscribe Photonic Professional GT system). In our case, the GalvoScanMode is used, for which an official smart algorithm called Galvo Perfect-Shape has been integrated into NanoWrite. In the continuous mode of GalvoScanMode, two consecutive and in-plane polylines are connected by a continuous and smooth trajectory, which reduces settling \& acceleration times and increases overall speed and trajectory accuracy. The laser intensity monitoring is synchronized with the galvo scanner, so that no laser intensity is emitted when bridging two lines.

It can be observed from Figure $2 b$ that, even with short segment of lines, the nonuniformity of width and height along the whole line is negligible. The elliptical trajectory-start and trajectoryend shapes are due to the intrinsic ellipsoid shape of the voxels. In addition, for printed bulk phase blocks in Figure 3j, the edge is quite smooth without defects. During the printing process of phase blocks, the hatching distance in $x y$ plance and slicing distance in $z$ direction were optimized. Thus 
the lines were written sufficiently close together, and the difference in index is so small that it doesn't affect the laser. The index is rather uniform both in the wavefront plane and along the propagation direction of laser.

Furthermore, the unwanted variation of scan speed changes the dwell time of femtosecond laser for the voxel and line, thus it can also be regarded as the laser power deviation. Measurements show that at wavelength around $633 \mathrm{~nm}$, the change of refractive index is less than 0.01 at different power intensities, which is equivalent to height deviation $n=0.017 \pi(10 \mathrm{~nm})$. According to the discussion in the part of fabrication defects on performance of Dammann gratings, the variation of refractive index can be treated as the second type of error, i.e., the effective height of fabricated phase blocks in DOEs is not exactly $580 \mathrm{~nm}$ as design (Figure 5b). As shown in Figure 5b, the refractive index variation has little to no effect on the beam spot array and increases the power ratio of the zero order spot by only $0.07 \%$. 
References

1. Guney, M.; Fedder, G. Estimation of Line Dimensions in 3D Direct Laser Writing Lithography. J. Micromech. Microeng. 2016, 26, 105011.

2. Saha, S. K.; Divin, C.; Cuadra, J. A.; Panas, R. M. Effect of Proximity of Features on the Damage Threshold during Submicron Additive Manufacturing via Two-Photon Polymerization. J. Micro. Nano-Manuf. 2017, 5, 031002.

3. Liu, Y.; Campbell, J.; Stein, O.; Jiang, L.; Hund, J.; Lu, Y. Deformation Behavior of Foam Laser Targets Fabricated by Two-Photon Polymerization. Nanomaterials 2018, 8, 498.

4. Jiang, L. J.; Zhou, Y. S.; Xiong, W.; Gao, Y.; Huang, X.; Jiang, L.; Baldacchini, T.; Silvain, J.F.; Lu, Y. F. Two-Photon Polymerization: Investigation of Chemical and Mechanical Properties of Resins Using Raman Microspectroscopy. Opt. Lett. 2014, 39, 3034-3037.

5. Oakdale, J. S.; Ye, J.; Smith, W. L.; Biener, J. Post-Print UV Curing Method for Improving the Mechanical Properties of Prototypes Derived from Two-Photon Lithography. Opt. Express 2016, 24, 27077-27086.

6. Ueno, K.; Juodkazis, S.; Shibuya, T.; Yokota, Y.; Mizeikis, V.; Sasaki, K.; Misawa, H. Nanoparticle Plasmon-Assisted Two-Photon Polymerization Induced by Incoherent Excitation Source. J. Am. Chem. Soc. 2008, 130, 6928-6929.

7. Sundaramurthy, A.; Schuck, P. J.; Conley, N. R.; Fromm, D. P.; Kino, G. S.; Moerner, W. Toward Nanometer-Scale Optical Photolithography: Utilizing the Near-Field of Bowtie Optical Nanoantennas. Nano Lett. 2006, 6, 355-360. 
8. Liu, Y.; Xiong, W.; Lu, Y.; Huang, X.; Liu, H.; Fan, L. S.; Jiang, L.; Silvain, J.-F.; Lu, Y. F. Precise Assembly and Joining of Silver Nanowires in Three Dimensions for Highly Conductive Composite Structures. Int. J. Extrem. Manuf. 2019, 1, 025001.

9. Sun, H.-B.; Suwa, T.; Takada, K.; Zaccaria, R. P.; Kim, M.-S.; Lee, K.-S.; Kawata, S. Shape Precompensation in Two-Photon Laser Nanowriting of Photonic Lattices. Appl. Phys. Lett. 2004, $85,3708-3710$.

10. Park, S.-H.; Kim, K. H.; Lim, T. W.; Yang, D.-Y.; Lee, K.-S. Investigation of ThreeDimensional Pattern Collapse Owing to Surface Tension Using an Imperfection Finite Element Model. Microelectron. Eng. 2008, 85, 432-439.

11. Ovsianikov, A.; Viertl, J.; Chichkov, B.; Oubaha, M.; MacCraith, B.; Sakellari, I.; Giakoumaki, A.; Gray, D.; Vamvakaki, M.; Farsari, M.; Fotakis, C. Ultra-Low Shrinkage Hybrid Photosensitive Material for Two-Photon Polymerization Microfabrication. ACS Nano 2008, 2, 2257-2262.

12. Purtov, J.; Verch, A.; Rogin, P.; Hensel, R. Improved Development Procedure to Enhance the Stability of Microstructures Created by Two-Photon Polymerization. Microelectron. Eng. 2018, $194,45-50$.

13. Arai, J. A Novel Non-Flammable Electrolyte Containing Methyl Nonafluorobutyl Ether for Lithium Secondary Batteries. J. Appl. Electrochem. 2002, 32, 1071-1079.

14. Park, J.-G.; Lee, S.-H.; Ryu, J.-S.; Hong, Y.-K.; Kim, T.-G.; Busnaina, A. A. Interfacial and Electrokinetic Characterization of IPA Solutions Related to Semiconductor Wafer Drying and Cleaning. J. Electrochem. Soc. 2006, 153, G811-G814.

15. Namatsu, H.; Yamazaki, K.; Kurihara, K. Supercritical Drying for Nanostructure Fabrication 
without Pattern Collapse. Microelectron. Eng. 1999, 46, 129-132.

16. Zandrini, T.; Liaros, N.; Jiang, L.; Lu, Y.; Fourkas, J.; Osellame, R.; Baldacchini, T. Effect of the Resin Viscosity on the Writing Properties of Two-Photon Polymerization. Opt. Mater. Express 2019, 9, 2601-2616.

17. Liu, Y.; Stein, O.; Campbell, J. H.; Jiang, L.; Petta, N.; Lu, Y. Three-Dimensional Printing and Deformation Behavior of Low-Density Target Structures by Two-Photon Polymerization. In Nanoengineering: Fabrication, Properties, Optics, and Devices XIV, SPIE Nanoscience + Engineering, San Diego, CA, USA, August 31, 2017; SPIE: Bellingham, WA, USA, 2017; p $103541 \mathrm{U}$.

18. Jiang, L.; Campbell, J.; Lu, Y.; Bernat, T.; Petta, N. Direct Writing Target Structures by TwoPhoton Polymerization. Fusion Sci. Technol. 2016, 70, 295-309. 